\title{
Índice de masa corporal, conductas alimentarias de riesgo y percepción de la imagen corporal en estudiantes de un colegio de bachilleres en Yucatán, México
}

\section{Body mass index, food risk and perception of body image in students of a bachelor's school in Yucatán, Mexico}

VEGA-MEDINA, Marian Cecilia†, HOIL-SANTOS, Jolly Josefina, CAMBRANES-PUC, Luis Humberto, OLIVA-PEÑA, Yolanda y ANDUEZA-PECH, María Guadalupe

Centro de Investigaciones Regionales Dr. Hideyo Noguchi. Universidad Autónoma de Yucatán. Av. Itzáes, Centro, 97000 Mérida, Yuc.

ID 1er Autor: Marian Cecilia, Vega-Medina

ID 1er Coautor: Jolly Josefina, Hoil-Santos / ORC ID: 0000-0002-0016-4879, Researcher ID Thomson: S-4756-2018, CVU CONACYT ID: 897371

ID 2do Coautor: Luis Humberto, Cambranes-Puc / ORC ID: 0000-0003-4124-5197, Researcher ID Thomson: W-50412019, CVU CONACYT ID: 372144

ID 3er Coautor: Yolanda, Oliva-Peña / ORC ID: 0000-0002-2396-8722, Researcher ID Thomson: S-5531-2018, CVU CONACYT ID: 77728

ID $4{ }^{\circ}$ Coautor: María Guadalupe, Andueza-Pech / ORC ID: 0000-0002-3083-0529, Researcher ID Thomson: S-4702-2018, CVU CONACYT ID: 84374

\section{Resumen}

La adolescencia se relaciona con cambios físicos y psicológicos y es un factor importante para el desarrollo de la imagen corporal. En esta etapa aumenta la conciencia del cuerpo debido a los cambios físicos, a la importancia que le brinda la sociedad a los rasgos físicos y a la creciente tendencia a compararse con las normas culturales; en adolescentes de ambos sexos existe la posibilidad de incrementos rápidos en la altura y el peso, pero en las mujeres, estos cambios corporales se asocian con mayor prevalencia de sobrepeso y obesidad; en la actualidad, el estilo de vida de las personas ha cambiado ya que dominan los malos hábitos alimenticios, el sedentarismo y el estrés y ha disminuido la actividad física. El objetivo del presente trabajo fue establecer la relación entre el IMC real, las conductas alimentarias de riesgo y la precepción de la imagen corporal en estudiantes de un colegio de bachilleres del estado de Yucatán. El estudio fue de corte transversal; participaron 212 estudiantes. Dos tercios de los participantes se percibieron con un cuerpo que no corresponde al suyo. Se encontró relación entre el IMC real y el IMC percibido, así como entre el IMC y la presencia de conductas alimentarias de riesgo.

Índice de Masa Corporal, conductas alimentarias de riesgo, percepción de la imagen corporal, adolescentes

\begin{abstract}
Adolescence is related to physical and psychological changes and is an important factor for the development of body image. At this stage, body awareness increases due to physical changes, society's importance to physical traits and the growing tendency to compare with cultural norms; in adolescents of both sexes there is the possibility of rapid increases in height and weight, but in women, these body changes are associated with a higher prevalence of overweight and obesity; At present, people's lifestyle has changed since bad eating habits, sedentary lifestyle and stress have dominated and physical activity has decreased. The objective of this work was to establish the relationship between the real BMI, risky eating behaviors and the perception of body image in students of a high school in the state of Yucatan. The study was cross-sectional; 212 students participated. Two thirds of the participants were perceived with a body that does not correspond to theirs. A relationship was found between real BMI and perceived BMI, as well as between BMI and the presence of risky eating behaviors.
\end{abstract}

Body Mass Index, risky eating behaviors, body image perception, adolescents

Citación: VEGA-MEDINA, Marian Cecilia, HOIL-SANTOS, Jolly Josefina, CAMBRANES-PUC, Luis Humberto, OLIVAPEÑA, Yolanda y ANDUEZA-PECH, María Guadalupe. Índice de masa corporal, conductas alimentarias de riesgo y percepción de la imagen corporal en estudiantes de un colegio de bachilleres en Yucatán, México. Revista de Ciencias de la Salud. 2019. 6-20: 8-13.

\footnotetext{
$\dagger$ Investigador contribuyendo como primer Autor
} 


\section{Introducción}

En la actualidad la transición epidemiológica, la muerte y las conductas de riesgo que se viven en los países desarrollados y en vías de desarrollo han cobrado mayor relevancia, incrementando la demanda no sólo del número de personas que requieren acceso a una buena atención en hospitales y consultorios, sino de la participación de diversas instancias o sectores donde los individuos sean provistos de herramientas que les permitan hacer frente a las situaciones que les enferman; enfocando diversas estrategias en la prevención y promoción del autocuidado de la salud, generando escenarios que permitan el desarrollo de estilos de vida saludable, sobre todo en aquellas poblaciones más vulnerables como son los adolescentes y adultos jóvenes (Saucedo y cols., 2010). La presión social a la que se ven sometidos estos grupos de población con la imposición del modelo estético de extrema delgadez hace que la preocupación por la imagen corporal haya crecido y lleve a conductas alimentarias de riesgo (Muñoz y cols., 2019).

La adolescencia es un período de transición entre la dependencia de la niñez y la independencia de la edad adulta. En ella, ocurren diferentes cambios biológicos, sociales y psicológicos que generan una sensación de perplejidad, dudas, miedos y ansiedad que la mayoría de las y los adolescentes superan con éxito. Es una etapa donde se esfuerzan por entenderse a sí mismos, exploran su propia identidad e intentan saber quiénes son, cómo son y hacia dónde se dirige su vida.

No solo tratan de comprenderse, sino también evalúan sus atributos, esto conforma su autoestima (Mora 2008); por ello es la etapa más vulnerable en relación con los problemas de imagen corporal, ya que es un momento en el ciclo vital caracterizado por diversos cambios, que provocan una mayor preocupación por la apariencia física. Valverde et al., (2010) subrayan que esta preocupación se torna diferente según el sexo, es decir, mientras los chicos se preocupan por una imagen corporal donde predomine la musculación, las chicas poseen ideales de belleza asociados a la delgadez e incluso, por debajo de una talla saludable.
De acuerdo con la encuesta intercensal 2015 la población en México continúa siendo predominantemente joven; $25.7 \% \quad$ (30.6 millones) de la población total son jóvenes de 15 a 29 años, $50.9 \%$ son mujeres y $49.1 \%$ son hombres. $35.1 \%$ son adolescentes de 15 a 19 años, $34.8 \%$ jóvenes de 20 a 24 años y 30.1\% tienen de 25 a 29 años. En Yucatán, la población registrada en 2010 fue 1,955,577 personas; un tercio del total de la población $(33,3 \%)$, es decir 654,860 personas tenía entre 0 y 17 años. El porcentaje de población masculina $(50.9 \%)$ fue ligeramente mayor que la femenina $(49,1 \%)$; $82,1 \%$ de niñas, niños y adolescentes vivían en poblaciones urbanas y 52,4\% fue de origen indígena (INEGI 2016). La Encuesta Nacional de Salud y Nutrición 2012, informó que, en el grupo de adolescentes, 29,9\% reflejaba sobrepeso y con obesidad, 12,2\%. La distribución por sexo mostró una prevalencia mayor para mujeres $(44,1 \%)$ en comparación con los hombres (42,7\%) (Oliva y cols. 2016). Una buena salud es considerada como el mejor recurso para el progreso personal, económico y social, y una dimensión importante de la calidad de vida del individuo, la familia y la comunidad (Saucedo y cols, 2010); el periodo adolescente es decisivo para la adquisición de estilos de vida, en el que tiene lugar la consolidación de algunos comportamientos provenientes de la infancia, así como la incorporación de otros nuevos adquiridos en sus contextos de socialización. La trascendencia de la adolescencia para el establecimiento del estilo de vida tiene que ver con los cambios psicológicos y contextuales que tienen lugar durante estos años en los que chicos $\mathrm{y}$ chicas realizan sus primeras salidas $\mathrm{y}$ reuniones con sus iguales sin la presencia de sus padres y en los que van ganando autonomía para tomar algunas decisiones relacionadas con el ocio y el estilo de vida por lo que conforme van avanzado en esta etapa, la influencia de los padres en la elección de alimentos para sus hijos va disminuyendo y la de sus iguales va en ascenso. Sin embargo, durante la adolescencia media (15-17 años) se produce un empeoramiento casi generalizado de las conductas de riesgo, ya que se inicia el consumo de tabaco y alcohol, comienzan las prácticas sexuales de riesgo, las chicas tienden a mostrarse descontentas con su imagen corporal y los chicos tienden a presentar problemas de adaptación a su entorno escolar y menor rendimiento académico, lo que puede llevarlos a experiencias de fracaso escolar y abandono académico (Hidalgo 2017). 
Hoy en día el estereotipo corporal raya en la delgadez extrema en las mujeres y la corpulencia por musculatura en los varones, estereotipos promovidos y vendidos por la industria y los medios de comunicación de manera exagerada. La figura corporal real, dista mucho de estos modelos estéticos dominantes lo que propicia malestar con la imagen corporal o incomodidad con respecto a ella, y que es generada por los mensajes, imágenes $\mathrm{y}$ comentarios emitidos por los medios de comunicación. La influencia de la publicidad hacia los jóvenes es inmensa, incluso basta con mirar a nuestro alrededor para encontrar algún anuncio donde no importando el producto promocionado se explota el cuerpo humano, presentando personas "bellas", principalmente mujeres, delgadas y casi siempre jóvenes. Lo anterior se traduce en la relación entre la sensibilidad (atención y reactividad) y la influencia o crítica cultural, entendiendo ésta como los mensajes verbales, imágenes relacionadas con el peso, la figura y la belleza emitidos por los medios de comunicación (Mora 2008).

Los jóvenes no están exentos de malos hábitos alimentarios y de poca actividad física, lo que está provocando en ellos cambios importantes en su peso y figura corporal, que pueden oscilar entre la obesidad y la desnutrición. En estudiantes universitarios, y en particular los que estudian nutrición influye mucho la presión sociocultural, lo que favorece la adopción de conductas alimentarias de riesgo, independientemente de su estatus nutricional (Díaz y cols., 2019).

Una herramienta útil y de bajo costo para la valoración antropométrica del estado nutricio es el índice de masa corporal (IMC); éste es un indicador del estado de nutrición que con mayor frecuencia es utilizado por su facilidad de estimación; es ampliamente estudiado como factor de riesgo asociado a los trastornos de conducta y de manera más específica con las conductas alimentarias, ya que se han encontrado importantes asociaciones (Lendechy 2019; Saucedo y cols., 2015)
Se ha demostrado que los individuos que presentan un IMC alto (sobrepeso u obesidad), tienden a alejar su imagen corporal de la "ideal"; y la influencia de factores socioculturales que han estigmatizado el sobrepeso y la obesidad los hace buscar estrategias para disminuir su peso corporal o modificar su figura, practicando conductas alimentarias de riesgo (CAR) (lendechy, 2019).

Ejemplo de lo anterior es un estudio realizado en estudiantes de 12 a 19 años del D.F. en donde se observó que aquellos que sobrestimaron su peso y, por lo tanto, su IMC, presentaron mayores prevalencias de CAR (Hidalgo, 2017). En otra investigación realizada por Saucedo y cols., (2015) en púberes mexicanos de ambos sexos, se encontró, a través de modelos predictivos, que a mayor IMC mayor seguimiento de conductas de riesgo y mayor influencia de factores socioculturales principalmente en mujeres.

Otro estudio fue el realizado por Lendechy y cols., (2019) en estudiantes de nutrición de una escuela privada de Mérida Yucatán, México con el objetivo de correlacionar el IMC autopercibido con el IMC real y su relación con la satisfacción del peso real para una detección temprana de alumnos con riesgo de desarrollar un trastorno alimenticio e implementar estrategias de prevención. Entre sus resultados encontraron un nivel de concordancia pobre de la autopercepción de la imagen corporal en los sujetos incluidos en el estudio; sin embargo, se observó que la mayoría de ellos señala que se sienten satisfechos con su imagen corporal actual.

Si bien es un hecho que la formación académica de los estudiantes de nutrición les dota de conocimientos básicos para identificar aquellas CAR que podrían ponerlos en riesgo de presentar un trastorno de la conducta alimentaria, ello no es suficiente para mantenerlos ajenos a la presión social por parte de los medios de comunicación, familiares y amigos para alcanzar el ideal de delgadez. 
Por ello se recomienda indagar en las causas que puedan motivar la insatisfacción corporal, el discurso de sus propios profesores sobre la forma física, el peso corporal y la ingesta de alimentos, así como las implicaciones emocionales y de desarrollo personal que pudieran orientar la aceptación de la propia figura corporal, para apoyar el diseño e implementación de programas de prevención de los trastornos de conducta alimentaria (Díaz y cols., 2019).

Tanto la distorsión como la insatisfacción con la imagen corporal (IC) son cruciales en el desarrollo de los trastornos alimentarios. La imagen corporal es "la imagen que se forma en la mente de nuestro propio cuerpo, es decir, el modo en que nuestro cuerpo se nos manifiesta". Por tanto, la imagen corporal no está, necesariamente, correlacionada con la apariencia física real; son claves las actitudes y valoraciones que el individuo hace de su propio cuerpo; está formada por diferentes componentes: el perceptual (percepción del cuerpo en su totalidad o de alguna de sus partes); el cognitivo (valoraciones respecto al cuerpo o una parte de éste); el afectivo (sentimientos o actitudes respecto al cuerpo o a una parte de éste y sentimientos hacia el cuerpo) y el conductual (acciones o comportamientos que se dan a partir de la percepción). La imagen corporal no es fija e inamovible, sino que puede ser diferente a lo largo de la vida; tiene mayor relevancia durante la adolescencia que es considerada de gran vulnerabilidad hacia la apariencia física ya que, en ésta, se desarrolla el sentido de la identidad y del rol sexual y es el periodo más sensible para el inicio de la insatisfacción corporal (Escandón y cols, 2019; Guadarrama y cols. 2018).

\section{Metodología}

El presente estudio fue de corte transversal; participaron 212 estudiantes de un Colegio de Bachilleres del estado de Yucatán, durante el periodo escolar 2018-2019, seleccionados mediante un muestreo de estimación de proporción; después de obtener el consentimiento informado se procedió a la medición antropométrica para la obtención del IMC de acuerdo a la clasificación de la OMS; posteriormente se aplicó un cuestionario para medir conductas alimentarias y una escala de evaluación de la imagen corporal.

\section{Resultados}

El estudio se realizó con un total de 212 estudiantes. El $48 \%(\mathrm{n}=101)$ fueron mujeres y el $52 \% \quad(n=111)$ fueron hombres. Las edades estuvieron en el rango entre 15 y 19 años con una media de 16.49 y desviación estándar de 0.873 .

Se determinó el IMC a través del peso y la talla de los estudiantes y se obtuvo que el $47.2 \%$ de ellos tuvieron un peso normal, el .5\% de los estudiantes estuvieron por debajo de su peso normal y el $52.3 \%$ estuvieron sobre su peso normal. Por sexo, el mayor porcentaje, tanto de mujeres como de hombres, se encontraron dentro de su peso normal; sin embargo, las mujeres presentaron un IMC por arriba de su peso normal $(53.46 \%)$ en mayor porcentaje respecto a los hombres $(51.35 \%)$ sin diferencia estadísticamente significativa $(\mathrm{p}>0.05)$.

En cuanto al IMC percibido en la muestra de estudiantes el $33.5 \%$ se percibió en su peso normal; sin embargo, podemos observar que el $37.7 \%$ de los estudiantes se percibieron con una figura superior a la real y el $28.8 \%$ con una inferior. Por sexo, el IMC percibido con mayor frecuencia en hombres fue el peso normal $(37.8 \%)$ y en mujeres, de la misma manera fue el peso normal, pero en menor porcentaje (28.7\%). Se observó una tendencia en las mujeres a identificarse con mayores estados de desnutrición en comparación a los hombres, la cual fue a estados de obesidad. La relación entre el sexo $y$ el IMC percibido no fue estadísticamente significativa $(\mathrm{p}>0.05)$.

Las conductas alimentarias de riesgo que se evaluaron en el estudio fueron conductas bulímicas, imagen corporal con tendencia a la delgadez, uso o abuso de laxantes, presencia de vómitos, restricción alimentaria, comer a escondidas y presión social percibida al aumentar de peso. Se consideró con riesgo a padecer conductas alimentarias de riesgo o tendencias a la realización de éstas a quien obtuvo una puntuación de 30 o mayor en el instrumento. El $83.5 \% \quad(n=177)$ no tuvieron riesgo de padecer conductas alimentarias de riesgo y el $16.5 \% \quad(n=35)$ tuvieron riesgo de padecer conductas alimentarias de riesgo. Por sexo se pudo observar que el $17.1 \%$ de los hombres tienen riesgo y el $15.8 \%$ de las mujeres, ligeramente mayor en los hombres, sin diferencias estadísticamente significativas. 
En la comparación entre el IMC real y el IMC percibido por los estudiantes se encontró que $34.43 \%$ de los estudiantes se percibieron de acuerdo con su IMC real y el $65.57 \%$ tuvieron una percepción inadecuada de la imagen corporal, $45.75 \%$ un IMC menor al real y $18.88 \%$ mayor al real, es decir la mayoría de los estudiantes se percibieron con un cuerpo que no correspondió a su IMC real, diferencia estadísticamente significativa $(\mathrm{p}=0.000)$, lo que sugiere que el IMC percibido está relacionado con el IMC real. Tanto hombres como mujeres se percibieron con un IMC menor al suyo.

En cuanto al IMC y las conductas alimentarias de riesgo se encontró una tendencia a presentar mayor riesgo de conductas alimentarias de riesgo cuando el IMC real estuvo por arriba del peso normal $(\mathrm{p}=0.009)$, lo que indica una significancia estadística; cabe señalar que, a pesar de que los hombres presentaron mayor riesgo de conductas alimentarias de riesgo, las mujeres presentaron mayor tendencia a desarrollarlas cuando el IMC real estuvo por arriba del peso normal.

\section{Conclusiones}

El hecho de que dos tercios de los estudiantes de un colegio de bachilleres se perciban con cuerpo que no corresponde al suyo muestra que se requieren más estudios que ayuden a comprender estos trastornos, sin embargo, reconocer que los grupos estudiantiles son una población de alto riesgo para desarrollar trastornos alimentarios y que dichos trastornos se encuentran estrechamente relacionados con la preocupación por su imagen corporal, es un elemento clave para el diseño de programas preventivos de trastornos alimentarios y de tamizaje de casos para su tratamiento oportuno.

Es necesario que las instituciones educativas y de salud difundan información acerca de los trastornos de conductas alimentarias como enfermedades y sus indicadores, las conductas alimentarias de riesgo, con enfoque en la población Juvenil, pues tienen gran riesgo de padecerlas, así como crear programas de activación física, realizar pláticas acerca de los hábitos alimenticios saludables, debido a la existencia de mayores niveles de sobrepeso y obesidad que desnutrición en esta institución educativa.

\section{Referencias}

Díaz Gutiérrez, M. C., Bilbao y Morcelle, G. M., Unikel Santoncini, C., Muñoz Espinosa, A., Escalante Izeta, E. I., \& Parra Carriedo, A. (2019). Relación entre estatus nutricional, insatisfacción corporal y conductas alimentarias de riesgo en estudiantes de nutrición. Revista mexicana de trastornos alimentarios, 10(1), 5365.

http://www.scielo.org.mx/scielo.php?script=sci _arttext\&pid=S2007-15232019000100053

Escandón-Nagel, N., Vargas, J. F., Herrera, A. C., \& Pérez, A. M. (2019). Imagen corporal en función de sexo y estado nutricional: Asociación con la construcción del sí mismo y de los otros. Revista mexicana de trastornos alimentarios, 10(1), 32-41.

http://www.scielo.org.mx/scielo.php?script=sci _arttext\&pid=S2007-

$15232019000100032 \& \operatorname{lng}=$ es\&nrm=iso\&tlng= es

Guadarrama, R. G., Navor, J. C. H., \& López, M. V. (2018). " Cómo me percibo y cómo me gustaría ser": un estudio sobre la imagen corporal de los adolescentes mexicanos. Revista de Psicología Clínica con Niños y Adolescentes, 5(1), 37-43.

Hidalgo-Rasmussen, C. A., Martín, H. S., \& Aguilera Cervantes, V. G. (2017). Percepción del peso corporal, comportamientos alimentarios y calidad de vida en estudiantes mexicanos. Revista Mexicana de Investigación en Psicología, 4(S1), 80-90.

Instituto Nacional de Estadística y Geografía (INEGI). (2016). Encuesta Intercensal EIC 2015. Base de datos. México. http://www3.inegi.org.mx/sistemas/saladeprens a/

Lendechy Maza, L. R., Cardeña Moreno, L. A., y Sansores España, D. (2019). Autopercepción y satisfacción de la imagen corporal y su concordancia con el IMC de la población estudiantil de la Licenciatura en Nutrición de una universidad privada. En: Perspectivas contextuales de salud y bienestar; Córdova G. M., León C. A. (Coords.). Editorial Qartuppi; Hermosillo, Sonora; 69-79. 
Mora, Z. S. (2008). Adolescencia e imagen corporal en la época de la delgadez. Reflexiones, 87(2), 8 .

Muñoz Pérez O., García Zamora P., Almeida Perales C. (2019). Factores determinantes de conductas alimentarias de riesgo y su prevalencia en estudiantes del área de ciencia de la salud. Revista electrónica semestral en Ciencias de la Salud; año 10, volumen 1; 1-14. http://revistas.uaz.edu.mx/index.php/ibnsina/art icle/view/504/476

Oliva, Y., Luna, M. O., Carvajal, A. M. S., Cárdenas, A. D. M., Pech, G. A., \& Castillo, I. A. G. (2016). Concordancia del IMC y la percepción de la imagen corporal en adolescentes de una localidad suburbana de Yucatán. Revista Biomédica, 27(2), 49-60.

Saucedo Molina T. D. J., Peña Irecta A., Fernández Cortés T. L., García Rosales A., Jiménez Balderrama R. E. (2010). Identificación de factores de riesgo asociados a trastornos de la conducta alimentaria y su relación con el Índice de Masa Corporal en universitarios del instituto de Ciencias de la Salud. Revista Médica de la Universidad Veracruzana, 10(2), 16-23. https://www.medigraphic.com/pdfs/veracruzana /muv-2010/muv102c.pdf

Saucedo-Molina, T. D. J., Rodríguez Jiménez, J., Oliva Macías, L. A., Villarreal Castillo, M., León Hernández, R. C., \& Fernández Cortés, T. L. (2015). Relación entre el índice de masa corporal, la actividad física y los tiempos de comida en adolescentes mexicanos. Nutrición Hospitalaria, 32(3), 1082-1090.

Valverde, P. R., De Los Santos, F. R., \& Rodríguez, C. M. (2010). Diferencias de sexo en imagen corporal, control de peso e Índice de Masa Corporal de los adolescentes españoles. Psicothema, 22(1), 77-83. 